\title{
Capacidade para o trabalho e intenção de saída da profissão na enfermagem de São Paulo
}

\author{
Work ability and intending to leave the nursing profession in São Paulo \\ Capacidad de trabajo e intención de dejar la profesión en enfermería en São Paulo
}

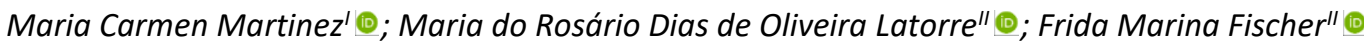

'WAF Informática e Saúde, São Paulo, SP, Brasil; "Universidade de São Paulo, São Paulo, SP, Brasil

\begin{abstract}
RESUMO
Objetivo: identificar o perfil de capacidade para o trabalho e intenção de saída da profissão na enfermagem. Método: um estudo transversal junto a 3051 profissionais do estado de São Paulo, com coleta de dados realizada por meio da internet avaliando características pessoais, ocupacionais, condições de trabalho, capacidade para o trabalho e ISP. Foi feita análise descritiva dos dados e associações verificadas por meio do teste qui-quadrado. Resultados: entre os profissionais, 55,4\% tinham capacidade para o trabalho comprometida e 33,5\% tinham intenção de saída presente. A intenção de saída aumentava na medida em que aumentava o comprometimento da capacidade para o trabalho $(p<0,001)$. Foram identificadas condições físicas e psicossociais inadequadas de trabalho. Conclusão: o comprometimento da capacidade para o trabalho e a presença de intenção de saída da profissão a foram frequentes entre os profissionais de enfermagem, sendo que condições laborais inadequadas estão presentes no trabalho desse contingente profissional.

Descritores: Saúde do Trabalhador; Trabalhador da Saúde; Avaliação da Capacidade de Trabalho; Condições de Trabalho.
\end{abstract}

\begin{abstract}
Objective: to identify the profile of work ability and intention to leave the nursing profession. Method: in this cross-sectional study of 3051 nurses in São Paulo state, data on personal and occupational characteristics, working conditions, work ability and intention to leave were collected through the Internet. Descriptive analysis was performed and associations were identified using the chi-square test. Results: $55.4 \%$ of the nurses' work ability was impaired and $33.5 \%$ intended to leave. Intention to leave increased as impairment of fitness for work increased $(p<0.001)$. Inadequate physical and psychosocial work conditions were identified. Conclusion: impaired work ability and the intention to leave the profession were frequent among professional nurses, and their work was found to suffer from inadequate labour conditions.

Descriptors: Occupational Health; Health Personnel; Work Capacity Evaluation; Working Conditions.

\section{RESUMEN}

Objetivo: identificar el perfil de capacidad para el trabajo e intención de dejar la profesión en enfermería. Método: estudio transversal junto a 3051 profesionales del estado de São Paulo, con recolección de datos realizada a través de internet, evaluando características personales y ocupacionales, condiciones de trabajo, capacidad para el trabajo e intención de abandonarlo. Se realizó un análisis descriptivo de los datos y asociaciones verificadas mediante la prueba de chi-cuadrado. Resultados: Entre los profesionales, el 55,4\% tenía capacidad de trabajo comprometida y el 33,5\% tenía presente intención de dejarlo. La intención de abandonarlo crecía a medida que aumentaba la discapacidad para el trabajo ( $p<0,001)$. Se identificaron condiciones de trabajo físicas y psicosociales inadecuadas. Conclusión: la disminución de capacidad para el trabajo y la presencia de intención de dejar la profesión fueron frecuentes entre los profesionales de enfermería; se presentan también condiciones de trabajo inadecuadas en el trabajo de este contingente profesional.

Descriptores: Salud Laboral; Personal de Salud; Evaluación de Capacidad de Trabajo; Condiciones de Trabajo.
\end{abstract}

\section{INTRODUÇÃO}

A enfermagem é elemento chave para o alcance dos objetivos de desenvolvimento sustentável e das metas de cobertura universal de saúde ${ }^{1}$. Entretanto, as condições de trabalho nessa profissão são caracterizadas por intensas cargas físicas e mentais, incluindo a intensificação da exposição a fatores psicossociais adversos ${ }^{1-4}$. Condições inadequadas de trabalho representam riscos para o comprometimento da capacidade para o trabalho (CT) e esse comprometimento pode ser seguido pelo incremento da intenção de saída da profissão (ISP) ${ }^{1-3}$.

CT é a condição que o trabalhador dispõe para executar seu trabalho, e é resultante das relações entre recursos individuais (capacidade física e mental, habilidades e conhecimento, estilos de vida, condições sociodemográficas e valores), demandas físicas, mentais e sociais do trabalho, e cultura organizacional e o ambiente de trabalho $0^{5-7} .0$ comprometimento da CT tem efeitos negativos, destacando-se adoecimento, insatisfação no trabalho, queda da produtividade, menor empregabilidade e saída precoce da profissão ${ }^{3,7}$.

Agradecimentos: ao Conselho Regional de Enfermagem do Estado de São Paulo- COREN SP, pelo apoio ao estudo

Autora correspondente: Maria Carmen Martinez, E-mail: mcmarti@uol.com.br

Editora Científica: Cristiane Helena Gallasch; Editora Associada: Magda Guimarães de Araujo Faria 
A ISP diz respeito ao intento de deixar a profissão, sendo preditor de uma decisão final de abandono da profissão ${ }^{3}$. A saída precoce da profissão pode resultar de fatores de pressão e de atração. Os fatores de pressão são aspectos adversos que levam as pessoas a quererem parar de trabalhar, tais como condições negativas no trabalho ou problemas de saúde. Os fatores de atração são incentivos como possibilidade de estudos, outra carreira ou regulamentação previdenciária ${ }^{3}$.

Estudos de seguimento e/ou com grandes grupos populacionais são realizados desde a década de 1990 junto à enfermagem na Europa, investigando as questões relativas à CT e ISP ${ }^{3}$. No Brasil, as pesquisas são pontuais e/ou em grupos específicos ${ }^{1,2,7}$ O país conta com mais de 2.400 .000 trabalhadores de enfermagem e $25,2 \%$ deles estão no estado de São Paulo ${ }^{8}$. Esse estudo objetiva identificar o perfil de CT e de ISP, além de descrever características individuais e estressores do ambiente físico e psicossocial do trabalho desse contingente laboral.

\section{MÉTOdo}

Estudo transversal junto a profissionais de enfermagem do estado de São Paulo. A coleta de dados ocorreu entre outubro de 2018 e março de 2019, quando havia 495.602 profissionais com inscrição ativa no Conselho Regional de Enfermagem de São Paulo (Coren-SP), distribuídos em 14 subseções regionais.

Foi critério de exclusão não ter endereço eletrônico válido cadastrado (cerca de $17,0 \%$ dos profissionais). A pesquisa foi disponibilizada para $100,0 \%$ dos 411.162 profissionais elegíveis. Desses, 13,3\% abriram a mensagem eletrônica enviada, 1,8\% acessaram o formulário e 1,0\% (3.993 pessoas) preencheram o formulário. Entre os respondentes, 942 (23,6\%) não eram ativos na profissão (embora com inscrição ativa no Coren-SP) e 3.051 (76,4\%) eram atuantes. Entre os não ativos, 9,1\% migraram para outras profissões/atividades, 10,7\% estavam desempregados, 2,1\% estavam afastados ou aposentados por doença/acidente/incapacidade/invalidez e 1,7\% eram aposentados por idade e/ou tempo de serviço. Os 3.051 respondentes ativos corresponderam a uma taxa de adesão de $0,74 \%$ em relação aos elegíveis. A análise das perdas mostrou maior proporção na amostra do que entre os não participantes quanto à faixa etária de menos de 40 anos $(50,1 \%$ vs $48,3 \%$, $p=0,046)$, residir na capital $(36,8 \%$ vs $35,0 \%, p=0,049)$ e ser enfermeiro $(61,6 \%$ vs $26,8 \%, p<0,001)$.

O Coren-SP enviou mensagem aos profissionais por meio de correio eletrônico contendo informações sobre a pesquisa e o link de acesso ao Formulário Google via Internet, e mais duas postagens estimulando a participação.

O primeiro módulo do formulário continha questões sobre características sociodemográficas (sexo, idade, estado conjugal, município de residência, renda familiar mensal, subseções do Coren-SP), estilo de vida (tabagismo, consumo de bebidas alcoólicas e questionário CAGE para avaliação de consumo alcoólico de risco, prática de atividade física e índice de massa corporal), histórico ocupacional (idade de ingresso na força de trabalho, tempo de trabalho, formação/titulação, situação de trabalho, vínculo contratual, área de atuação, cargo/função, segundo emprego, turno de trabalho, jornada de trabalho semanal, história recente de doença/acidente no trabalho).

O segundo módulo iniciou com a versão brasileira do ICT - Índice de Capacidade para o Trabalho, fornecendo um escore variando de 7 a 49 pontos, a partir de 7 dimensões: CT atual e comparada com a melhor de toda a vida; CT em relação às exigências do trabalho; número atual de doenças diagnosticadas por médico; perda estimada para o trabalho devido a doenças; falta ao trabalho por doenças; prognóstico próprio sobre a CT; e recursos mentais ${ }^{6,10}$. 0 escore foi categorizado em CT em ótima, boa, moderada ou baixa' e a variável foi dicotomizada em CT comprometida (moderada ou baixa) e preservada (ótima ou boa) $)^{5,7,9}$.

A ISP foi avaliada com base na questão do NEXT-Study "Com que frequência durante os últimos 12 meses você

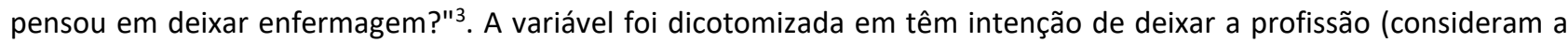
possibilidade diversas vezes no mês ou mais frequentemente) e não têm intenção de deixar a profissão (consideram a possibilidade algumas vezes por ano ou nunca) $)^{3}$.

Para avaliar risco para desgaste psicossocial no trabalho utilizou-se a Escala Estresse no Trabalho, adaptação para uso no Brasil do Job Content Questionnaire, baseado no Modelo Demanda-Controle ${ }^{10}$. A escala tem 17 questões abrangendo as dimensões demandas, controle e apoio social ${ }^{10}$. As dimensões demanda e controle foram dicotomizadas pelo ponto médio de cada escore, e combinadas em 4 categorias: trabalho com elevado desgaste, trabalho ativo, trabalho com baixo desgaste e trabalho passivo ${ }^{7,10}$. A dimensão apoio social no trabalho foi dicotomizada pelo ponto médio do escore.

Os estressores do ambiente psicossocial do trabalho foram avaliados por meio da versão brasileira do questionário Desequilíbrio Esforço-Recompensa, estruturado sobre o modelo teórico de mesmo nome e composto por 17 questões abrangendo 3 dimensões: esforços, recompensas e excesso de comprometimento ${ }^{10}$. Para classificação do desequilíbrio é calculada a razão entre esforço e recompensa e multiplicada por $6 / 11$, fornecendo um coeficiente variando de 0,17 a 5,00 pontos, e esse escore foi categorizado em tercis ${ }^{10}$. O excesso de comprometimento foi dicotomizado por meio do ponto médio do escore. 
Para avaliar características do ambiente físico que pudessem contribuir para o desenvolvimento de agravos musculoesqueléticos foi utilizada a versão validada para uso no Brasil do questionário Atividades Relacionadas Ao Trabalho Que Podem Contribuir Para Dor e/ou Lesão, fornecendo um escore de 0 a 150 pontos ${ }^{10}$. Os escores foram categorizados em tercis.

A qualidade do sono e a presença de insônia foram avaliadas por meio da versão em português do Questionário de sono Karolinska11.

A confiabilidade dos questionários foi investigada por meio do coeficiente Alfa de Cronbach. Foi feita análise descritiva e associações foram verificadas por meio do teste qui-quadrado e o nível descritivo adotado foi $p<0,050$.

O estudo foi aprovado pela Diretoria do Coren-SP. Os pesquisadores não tiveram acesso às bases de dados cadastrais ou a identificação nominal dos profissionais, aderindo às premissas de segurança da informação do CorenSP. O estudo foi aprovado pelo Comitê de Ética e Pesquisa da Faculdade de Saúde Pública da Universidade de São Paulo (no 2.614.513) e foi realizado de acordo com os princípios da Declaração de Helsinki e da Associação Médica Mundial. A participação dos trabalhadores foi voluntária, mediante Termo de Consentimento Livre e Esclarecido, e a confidencialidade dos dados individuais foi garantida.

\section{RESULTADOS}

As características qualitativas sociodemográficas e do estilo de vida estão apresentadas na Tabela 1.

TABELA 1: Análise descritiva da população de estudo segundo características demográficas. São Paulo, SP, Brasil, 2019.

\begin{tabular}{|c|c|c|c|}
\hline Variável & & $\mathbf{n}$ & $\%$ \\
\hline \multirow[t]{3}{*}{ Sexo } & Feminino & 2.612 & 85,6 \\
\hline & Masculino & 436 & 14,3 \\
\hline & Não quero responder & 3 & 0,1 \\
\hline \multirow[t]{5}{*}{ Faixa etária (em anos) } & Até 30,0 & 476 & 15,6 \\
\hline & 31,0 a 40,9 & 1.180 & 38,7 \\
\hline & 41,0 a 50,9 & 797 & 26,1 \\
\hline & 51,0 a 60,9 & 513 & 16,8 \\
\hline & 61,0 e mais & 85 & 2,8 \\
\hline \multirow[t]{3}{*}{ Estado conjugal } & Casado(a) ou com companheiro(a) & 1.857 & 60,9 \\
\hline & Separado(a) ou divorciado(a) ou viúvo(a) & 408 & 13,4 \\
\hline & Solteiro(a) & 786 & 25,8 \\
\hline \multirow[t]{6}{*}{ Renda familiar mensal (em no de salários-mínimos) } & 10,1 e mais & 297 & 9,7 \\
\hline & 7,1 a 10,0 & 509 & 16,7 \\
\hline & 5,1 a 7,0 & 541 & 17,7 \\
\hline & 3,1 a 5,0 & 881 & 28,9 \\
\hline & até 3,0 & 744 & 24,4 \\
\hline & Prefiro não responder & 79 & 2,6 \\
\hline \multirow[t]{3}{*}{ Subseção } & São Paulo - Capital & 1.124 & 36,8 \\
\hline & Campinas & 462 & 15,1 \\
\hline & Demais & 1.465 & 48,0 \\
\hline \multirow[t]{2}{*}{ Tabagismo } & Não fumo & 2.769 & 90,8 \\
\hline & Sim, fumo & 282 & 9,2 \\
\hline \multirow[t]{3}{*}{ Consumo de bebidas alcoólicas } & Nunca ou raramente & 1.738 & 57,0 \\
\hline & Eventualmente & 1.201 & 39,4 \\
\hline & Frequentemente & 112 & 3,7 \\
\hline \multirow[t]{2}{*}{ Perfil indicativo de consumo alcoólico de risco } & Não & 2.916 & 95,6 \\
\hline & Sim & 135 & 4,4 \\
\hline \multirow[t]{2}{*}{ Atividade física regular nos últimos 12 meses } & Sim & 1.298 & 42,5 \\
\hline & Não & 1.753 & 57,5 \\
\hline \multirow[t]{5}{*}{ IMC - Índice de massa corporal } & Desnutrição & 34 & 1,1 \\
\hline & Eutrofia & 981 & 32,2 \\
\hline & Sobrepeso & 1.080 & 35,4 \\
\hline & Obesidade & 918 & 30,1 \\
\hline & Não informado & 38 & 1,2 \\
\hline \multirow[t]{3}{*}{ Qualidade do sono / distúrbios do sono } & Boa & 1.655 & 54,2 \\
\hline & Intermediária & 880 & 28,8 \\
\hline & Ruim & 516 & 16,9 \\
\hline \multirow[t]{2}{*}{ Insônia } & Sem sintomas de insônia & 1.135 & 37,2 \\
\hline & Com sintomas de insônia & 1.916 & 62,8 \\
\hline Total & & 3.051 & 100,0 \\
\hline
\end{tabular}


A maior parte dos participantes foram mulheres (85,6\%) e pessoas casadas/com companheiro/a (60,9\%). Os participantes eram adultos jovens, sendo 54,3\% tinham idade inferior a 41,0 anos. Renda familiar mensal de até 5,0 salários-mínimos foi informada por $53,3 \%$ dos trabalhadores. As Subseções com maior percentual de participantes foram São Paulo $(36,8 \%)$ e Campinas (15,1\%). Observou-se que 90,8\% negaram tabagismo. A maioria não teve consumo rotineiro de bebidas alcoólicas (57,0\%), mas 4,4\% dos participantes mostraram consumo de risco; 57,5\% informaram prática de atividade física regular, 35,4\% tinham sobrepeso e 30,0\% eram obesos. Quanto ao sono, 45,8\% reportaram qualidade do sono intermediária/ruim decorrente de distúrbios do sono e 62,8\% reportaram sintomas de insônia.

A Tabela 2 apresenta os resultados das características ocupacionais.

Tabela 2: Análise descritiva da população de estudo segundo características ocupacionais. São Paulo, SP, Brasil, 2019.

\begin{tabular}{|c|c|c|}
\hline Variável & $\mathbf{n}$ & $\%$ \\
\hline \multicolumn{3}{|l|}{ Idade com que começou a trabalhar (anos) } \\
\hline Menor que 14,0 & 330 & 10,8 \\
\hline 14,0 a 17,9 & 1.165 & 38,2 \\
\hline 18,0 ou mais & 1.556 & 51,0 \\
\hline \multicolumn{3}{|l|}{ Anos de trabalho na profissão atual } \\
\hline Menos que 6,0 & 430 & 14,1 \\
\hline 6,0 a 10,9 & 693 & 22,7 \\
\hline 11,0 a 15,9 & 576 & 18,9 \\
\hline 16,0 ou mais & 1.352 & 44,3 \\
\hline \multicolumn{3}{|l|}{ Categoria profissional } \\
\hline Enfermeiro & 1.879 & 61,6 \\
\hline Técnico de enfermagem & 993 & 32,5 \\
\hline Auxiliar / Atendente de enfermagem & 179 & 5,9 \\
\hline \multicolumn{3}{|l|}{ Principal vínculo empregatício na enfermagem nos últimos 12 meses } \\
\hline Empregado de instituição privada com registro em carteira de trabalho & 1.497 & 49,1 \\
\hline Funcionário público & 1.151 & 37,7 \\
\hline Outras & 403 & 13,2 \\
\hline \multicolumn{3}{|l|}{ Principal área de atuação na enfermagem nos últimos 12 meses } \\
\hline Hospital & 1.444 & 47,3 \\
\hline Unidade Básica de Saúde, Ambulatórios ou assemelhados & 660 & 21,6 \\
\hline Pronto Socorro, Serviços de emergência ou assemelhados & 315 & 10,3 \\
\hline Instituição de ensino & 146 & 4,8 \\
\hline Serviços domiciliares ou assemelhados & 105 & 3,4 \\
\hline Outros & 381 & 12,5 \\
\hline \multicolumn{3}{|l|}{ Principal cargo/função na enfermagem nos últimos 12 meses } \\
\hline Prestação de cuidados diretos ao paciente / cliente & 2.025 & 66,4 \\
\hline Chefia / liderança & 596 & 19,5 \\
\hline Ensino / pesquisa & 154 & 5,0 \\
\hline Outros & 176 & 9,0 \\
\hline \multicolumn{3}{|l|}{ Acidente ou doença relacionada ao trabalho nos últimos 12 meses } \\
\hline Não & 2.100 & 68,8 \\
\hline Sim & 951 & 31,2 \\
\hline \multicolumn{3}{|l|}{ Segundo emprego nos últimos 12 meses } \\
\hline Não & 1.977 & 64,8 \\
\hline Sim & 1.074 & 35,2 \\
\hline \multicolumn{3}{|l|}{ Trabalho noturno (10 e/ou 2o emprego) } \\
\hline Não & 2.164 & 70,9 \\
\hline Sim & 887 & 29,1 \\
\hline \multicolumn{3}{|l|}{ Carga horária semanal total - emprego e doméstica (em horas) } \\
\hline Até 39,9 & 151 & 4,9 \\
\hline 40,0 a 49,9 & 419 & 13,7 \\
\hline 50,0 a 59,0 & 567 & 18,6 \\
\hline 60,0 a 69,0 & 677 & 22,2 \\
\hline 70,0 a 79,0 & 569 & 18,6 \\
\hline 80 ou mais & 668 & 21,9 \\
\hline Total & 3.051 & 100,0 \\
\hline
\end{tabular}


Entre os trabalhadores, $49,0 \%$ iniciaram antes dos 18,0 anos e $44,3 \%$ tinham 16,0 ou mais anos na profissão. Entre os participantes, $61,6 \%$ eram enfermeiros, $32,5 \%$ técnicos de enfermagem e 5,9\% auxiliares de enfermagem. Os vínculos empregatícios predominantes foram as instituições privadas com registro em carteira de trabalho (49,1\%) e o funcionalismo público (37,7\%). As principais áreas de atuação foram o setor hospitalar (47,3\%) e a a atenção básica à saúde (21,6\%). Os cargos/funções predominantes foram a prestação de cuidados diretos ao paciente/cliente $(66,4 \%)$ e a chefia/liderança (19,5\%). Acidente/doença relacionada ao trabalho nos últimos 12 meses foi relatada por $31,2 \%$ dos profissionais. Entre os profissionais, 35,2\% tinham mais de um vínculo de trabalho e 29,1\% atuavam em horário noturno, sendo a carga semanal total (emprego mais doméstica) igual ou maior a 60,0 horas entre 62,7\% dos participantes.

Na Figura 1 observa-se que as situações de trabalho inadequadas foram mais frequentes quanto às altas demandas psicossociais (86,8\%), excesso de comprometimento elevado $(45,1 \%)$, exposições elevadas em atividades que favorecem a ocorrência de dor ou lesão $(36,2 \%)$ e desequilíbrio entre esforços e recompensas $(31,6 \%)$.

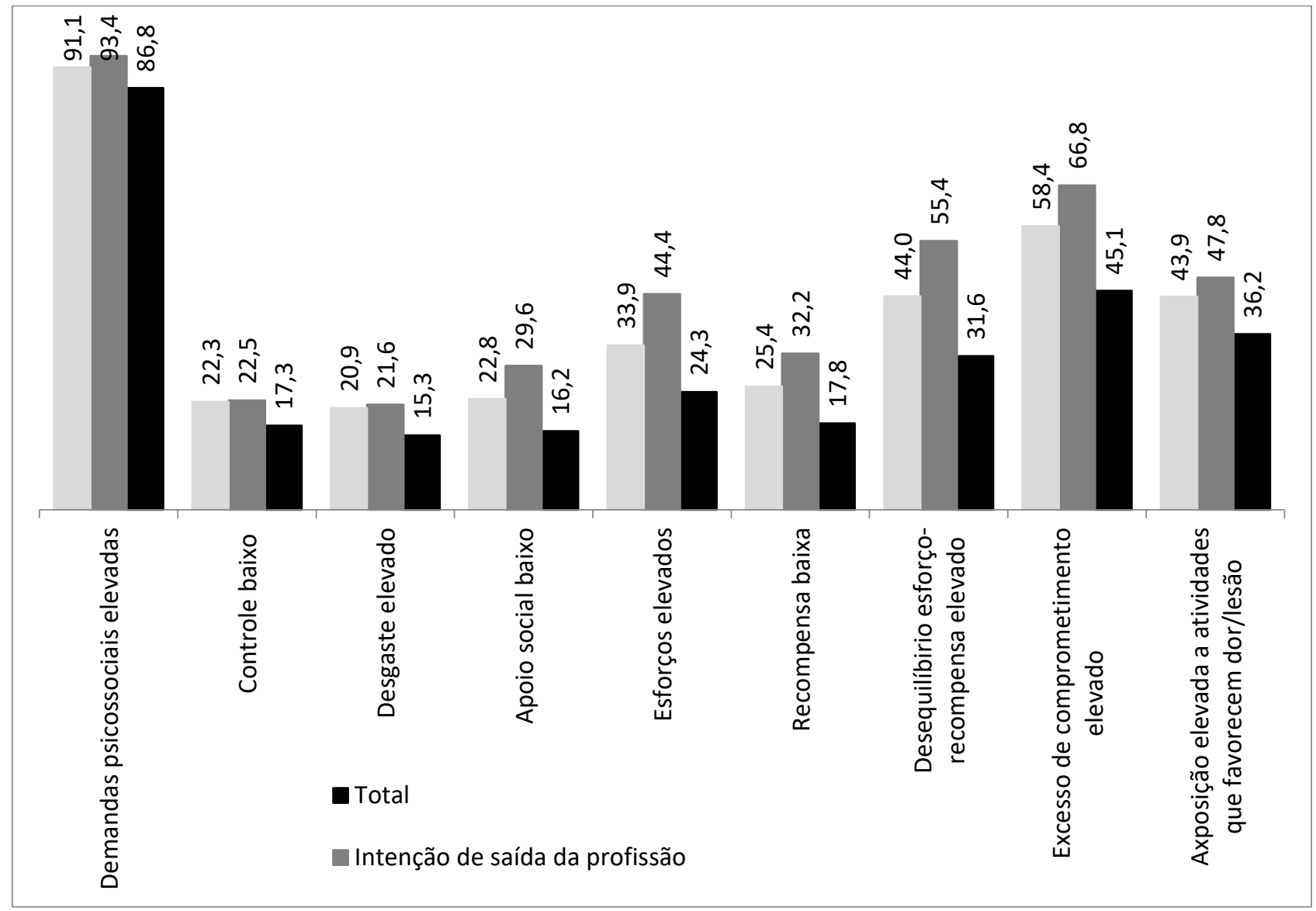

FIGURA 1: Frequência (\%) de exposição aos estressores do ambiente psicossocial e das condições de trabalho segundo presença de intenção de saída da profissão, presença de comprometimento da capacidade para o trabalho e para o total dos participantes, Enfermagem (n=3.051). São Paulo, 2019.

Houve exposição mais frequente entre enfermeiros e técnicos de enfermagem quanto às altas demandas de trabalho $(p=0,008)$; exposição mais frequente entre técnicos e auxiliares de enfermagem quanto ao baixo controle $(p<0,001)$, ao alto desgaste $(p<0,001)$ e à exposição elevada e moderada a situações que favorecem a ocorrência de dor ou lesão $(p<0,001)$; e exposição mais frequente entre os enfermeiros quanto ao desequilíbrio esforço-recompensa $(p=0,004)$ e ao alto excesso de comprometimento $(p<0,001)$.

Os agravos à saúde mais frequentes referidos foram: doença musculoesquelética nas costas, braços, pernas ou outra parte $(62,9 \%)$, distúrbio mental leve $(59,3 \%)$, lesão decorrente de acidente $(58,9 \%)$, doença metabólica/endócrina $(42,8 \%)$, doença respiratória (39,8\%), doença neurológica ou dos órgãos dos sentidos (39,6\%), e doença/problema digestivo (38,8\%).

Entre os participantes, $11,0 \%$ apresentaram CT ótima, 33,6\% boa, 36,4\% moderada e 19,0\% baixa, indicando que $55,4 \%$ tinham CT comprometida. Enfermeiros apresentaram CT comprometida com menor frequência (51,7\%) do que técnicos $(60,7 \%)$ e auxiliares $(64,2 \%)(p<0,001)$. Quanto à ISP, $33,7 \%$ dos participantes nunca pensaram em deixar a 
enfermagem, 32,5\% algumas vezes por ano, 12,1\% algumas vezes por mês, 9,7\% algumas vezes por semana, $11,7 \%$ pensaram todos os dias e $0,2 \%$ não responderam, apontando que 33,5\% dos trabalhadores pensaram frequentemente em deixar a enfermagem. A ISP não foi diferente entre as categorias profissionais $(p=0,128)$.

Houve associação entre CT e ISP $(p<0,001)$, ocorrendo aumento da ISP à medida que diminuía a CT: 11,0\% entre os trabalhadores com ótima CT, 20,6\% entre aqueles com boa CT, 38,4\% entre aqueles com moderada CT e $60,1 \%$ entre aqueles com baixa CT. A Figura 1 ilustra que, entre os profissionais com CT comprometida e entre aqueles com presença de ISP, a exposição a fatores psicossociais e condições de trabalho inadequadas foi mais frequente do que entre o conjunto dos participantes ( $p<0,001$ para todas as variáveis).

\section{DISCUSSÃO}

Observou-se elevada frequência de profissionais de enfermagem com a CT comprometida e com ISP. Também houve exposição moderada ou intensa aos FPST, em especial às demandas psicossociais, que representam riscos para ocorrência de estresse, adoecimento, incapacidade laboral e intenção de saída da profissão. Esses resultados apontam para um quadro preocupante na força de trabalho da enfermagem no estado de São Paulo.

Houve predominância do sexo feminino, de jovens, de pessoas casadas/com companheiro, com baixa renda familiar e residentes na subseção São Paulo, perfil esse que é característico da profissão. $O$ trabalho na enfermagem é historicamente desvalorizado e permeado por questões de gênero, o que se traduz em condições laborais inadequadas, precarização do trabalho, baixa remuneração e responsabilidade pelo sustento familiar parcial ou completo ${ }^{1,7}$.

Houve baixa frequência de tabagismo e de consumo alcoólico de risco, porém sobrepeso/obesidade e sedentarismo foram frequentes, situações que podem ser favorecidas por condições do trabalho inadequadas, resultando em doenças crônicas e comprometimento da $\mathrm{CT}^{7}$.

Parcelas importantes dos trabalhadores começaram a trabalhar com pouca idade e estavam há vários anos na profissão, indicando exposição a riscos laborais por longo tempo. Os cargos/funções predominantes foram a prestação de cuidados diretos ao paciente/cliente e cargos de chefia/liderança em nível intermediário. A efetivação dos processos de trabalho da enfermagem se dá por meio de estruturas hierarquizadas verticalmente, com divisão e fragmentação das atividades e das responsabilidades, onde prioritariamente técnicos e auxiliares de enfermagem desempenham tarefas assistenciais e enfermeiros estão em atividades de gestão ${ }^{12}$.

Observaram-se longas e frequentes duplas jornadas de trabalho, incluindo trabalho noturno. Estas situações estão associadas a desfechos negativos como fadiga, adoecimento, distúrbios do sono, comprometimento dos relacionamentos sociais/familiares, queda de produtividade e erros assistenciais ${ }^{3,15}$. Grande parcela dos trabalhadores informou sintomas de insônia e qualidade de sono intermediária ou ruim, e que podem ser decorrentes tanto da exposição a ambiente psicossocial inadequado como das jornadas de trabalho noturnas ou em turnos ${ }^{7,11}$.

Foram observadas exposições a situações inadequadas de trabalho, incluindo fatores do ambiente psicossociais e físico. Essas características do trabalho favorecem a ocorrência do sofrimento mental, desgaste e adoecimento ${ }^{1,3,4,7}$. Esses resultados foram corroborados pela ocorrência de história recente de acidente ou doença relacionada ao trabalho e de agravos autorreferidos (agravo musculoesquelético, lesões decorrentes de acidente e distúrbios mentais). Os esforços físicos nas atividades da enfermagem são intensos, muitas vezes potencializados pela indisponibilidade de recursos materiais adequados ${ }^{3,4}$. Destaca-se também o crescente adoecimento mental na enfermagem, vinculado à exposição a situações inadequadas do ambiente psicossocial do trabalho, aumento das exigências e precarização do trabalho ${ }^{1,2,7,10}$. Em recente estudo, 52,0\% dos profissionais de enfermagem informaram ter sofrido adoecimento mental (em especial ansiedade, depressão e esgotamento), decorrentes principalmente da sobrecarga de trabalho, das condições de trabalho e do clima organizacional ${ }^{8}$.

Nessa pesquisa, mais da metade dos profissionais de enfermagem apresentou CT comprometida. Mesmo considerando diferenças metodológicas e populacionais, o resultado apontou para um perfil mais preocupante do que outros estudos brasileiros, com valores próximos a $40,0 \%{ }^{14,15}$. Um estudo com enfermeiros da área hospitalar de 10 países da Europa, evidenciou CT comprometida em $23,0 \%$ das participantes ${ }^{3}$. O comprometimento da CT se manifesta na forma de dificuldades e impedimentos que o trabalhador percebe ao desenvolver suas atividades laborais ${ }^{3,6,7}$.

O lugar que o trabalhador ocupa na organização do trabalho vai determinar o que e como ele vai realizar suas atividades, condicionando diferentes formas de exposição aos riscos e diferentes formas de adoecimento. Os enfermeiros apresentam CT comprometida com menor frequência do que os técnicos e auxiliares de enfermagem. Esse comportamento foi observado em outros estudos, dado que a divisão do trabalho de enfermagem faz com aqueles executando tarefas com maiores cargas físicas apresentem adoecimento mais intenso e precoce ${ }^{7,14}$. 
Cerca de um terço dos profissionais mostraram ISP, sem diferenças entre as categorias profissionais. Essa frequência foi superior à observada em outros estudos brasileiros e na Europa, com variações de $16,0 \%$ a $24,9 \%{ }^{1-3}$. A ISP na enfermagem é um problema de saúde pública quando se considera a relevância desses profissionais na gestão e nas práticas assistenciais, a distribuição regional desigual e a demanda crescente decorrente do envelhecimento populacional ${ }^{1}$.

Esse estudo mostrou uma forte associação entre CT e ISP, sendo que a literatura evidencia que trabalhadores com comprometimento da CT têm maior risco para deixar o trabalho ${ }^{2,3}$. A decisão de deixar a profissão de enfermagem é precedida da intenção de sair, que por sua vez é influenciada por uma série de fatores subjacentes, incluindo aspectos negativos das condições e da organização do trabalho e situações pessoais e macrossociais, além de comprometimento da saúde e da $\mathrm{CT}^{1,3}$.

Os estressores psicossociais e físicos do trabalho identificados nesse estudo ocorreram mais frequentemente de forma inadequada entre os trabalhadores com CT comprometida e com ISP. O trabalho físico e/ou mental extenuante aumenta a susceptibilidade ao adoecimento, com consequente limitação na CT e levam os trabalhadores a querer deixar seu trabalho na configuração em que se encontra, predizendo a ISP e contribuindo para a saída do emprego voluntária ou não ${ }^{3,7}$. Outros estudos apontam a discrepância entre más condições de trabalho e CT, favorecendo a ISP, destacamse as condições insatisfatórias de valorização e de oportunidades de desenvolvimento, demandas emocionais excessivas e cargas físicas desgastantes e experiência de exposição à violência ${ }^{1-3}$.

Os resultados desse estudo exploratório fornecem um perfil da situação e apontam implicações para políticas públicas e de gestão institucional no sentido de promover a CT e prevenir a saída da profissão. O ganho desse estudo é ser o primeiro com abrangência estadual (São Paulo), contemplando as diferentes categorias profissionais da enfermagem e suas áreas de atuação, mostrando as características do problema (comprometimento da CT e ISP).

A opção de coleta de dados via Internet se deu em função da facilidade operacional em um estudo de abrangência estadual. A taxa de resposta foi de $0,74 \%$, próxima à expectativa de efetividade do COREN-SP para as ações de $e$-mail marketing ${ }^{13}$, com maior participação de enfermeiros do que de técnicos e auxiliares. Cabe destacar que a baixa adesão não elimina a contribuição que um estudo com voluntários pode oferecer para aprofundar a compreensão de eventos clínicos e sociais e disponibilizar informações relevantes ${ }^{16}$.

Em que pesem iniciativas institucionais que vêm sendo desenvolvidas em prol da enfermagem ${ }^{8}$, ainda há muito que fazer para garantir que essa força de trabalho esteja saudável, capacitada e disponível para atender às necessidades populacionais. Questões como promoção da CT, prevenção da ISP e melhoria das condições e da organização do trabalho, incluindo adequação de cargas de trabalho, proteção contra riscos laborais, valorização profissional e remuneração adequada, devem fazer parte das políticas públicas e institucionais, com diferentes tipos de intervenção, adequadas às necessidades e expectativas de cada situação $0^{1-3,7,12}$.

\section{CONCLUSÃO}

O comprometimento da CT e a ISP na Enfermagem de São Paulo apresentaram percentuais elevados. Esses profissionais frequentemente atuam em ambientes e condições inadequadas de trabalho, em especial no que diz respeito a demandas psicossociais elevadas, desequilíbrio entre esforços envidados e recompensas recebidas no emprego, elevado desgaste no trabalho, situações que favorecem a ocorrência de dor/lesão musculoesquelética, e excesso de comprometimento no trabalho.

\section{REFERÊNCIAS}

1. Oliveira DR, Griep RH, Portela LF, Rotenberg L. Intention to leave profession, psychosocial environment and self-rated health among registered nurses from large hospitals in Brazil: a cross-sectional study. BMC Health Serv. Res. [Internet]. 2017 [cited 2021 Feb 23]; 17:21. DOI: https://doi.org/10.1186/s12913-016-1949-6.

2. Bordignon M, Monteiro MI. Predictors of nursing workers' intention to leave the work unit, health institution and profession. Rev. Lat. Am. Enfermagem [Internet], 2019 [cited 2021 Feb 23]; 27:e3219. DOI: https://doi.org/10.1590/1518-8345.3280.3219.

3. Hasselhorn H-M, Müller BH, Tackenberg P, editors. NEXT Scientific Report - July 2005. Wuppertal: University of Wuppertal. 2005 [cited 2021 Jun 14]. Available from:

https://www.researchgate.net/publication/260592268_Next_Scientific_Report_July_2005.

4. Martinez MC, Latorre M do RD de O, Fischer FM. Stressors influence work ability in different age groups of nursing professionals: 2-year follow-up. Ciênc Saúde Coletiva [Internet]. 2017 [cited 2021 Feb 23]; 22(5):1589-600. DOI: https://doi.org/10.1590/1413-81232017225.09682015.

5. Smyth J, Pit Sabrina Winona, Hansen V. Can the work ability model provide a useful explanatory framework to understand sustainable employability amongst general practitioners: a qualitative study. Hum. Resour. Health [Internet]. 2018 [cited 2021 Jun 14]; 16(1):32. DOI: https://doi.org/10.1186/s12960-018-0292-x. 
6. Ilmarinen J. From work ability research to implementation. Int. J Environ. Res. Public Health. [Internet]. 2019 [cited 2021 Feb 23]; 16(16):2882. DOI: https://doi.org/10.3390/ijerph16162882.

7. Martinez MC, Fischer FM. Work Ability as Determinant of Termination of Employment. To Resign or Be Dismissed? J. Occup. Environ. Med. [Internet]. 2019 [cited 2021 Feb 23]; 61(6):e272-81. DOI: https://doi.org/10.1097/JOM.0000000000001599.

8. Coren - Conselho Regional de Enfermagem de São Paulo [site de Internet]. 2021 [cited 23 Fev 2021]. Available from: https://portal.coren-sp.gov.br/institucional/.

9. Martinez MC, Latorre MRDO, Fischer FM. Stressors influence work ability in different age groups of nursing professionals: 2year follow-up. Ciênc. Saúde Colet. [Internet]. 2017 [cited 2021 Jun 14]; 22(5):1589-600. DOI: https://doi.org/10.1590/141381232017225.09682015.

10. Martinez MC, Fischer FM. Psychosocial factors at hospital work: experienced conditions related to job strain and effort-reward imbalance. Rev. Bras. Saúde Ocup. [Internet]. 2019 [cited 2021 Jun 14]; 44:e12. DOI: https://doi.org/10.1590/23176369000025918.

11. Vasconcelos SP, Fischer FM, Reis AOA, Moreno CRC. Factors associated with work ability and perception of fatigue among nursing personnel from Amazonia. Rev. Bras. Epidemiol. [Internet]. 2011 [cited 2021 Feb 23]; 14(4):688-97. DOI: https://doi.org/10.1590/S1415-790X2011000400015.

12. Leal LA, Henriques SH, Brito LSS, Celestino LC, Ignácio DS, Silva AT. Health care models and their relationship with hospital nursing management. Rev Enferm UERJ [Internet]. 2019 [cited 2021 Feb 23]; 27:e43769. DOI: https://doi.org/10.12957/reuerj.2019.43769.

13. Hagopian EM. Contours of violence in nursing: quantitative approach [Doctoral dissertation]. São Paulo: Escola de Enfermagem da Universidade de São Paulo. 2019 [cited 2021 Feb 23]. DOI: https://doi.org/10.11606/T.7.2020.tde-11122019-134040.

14. Magnago TSBS, Prochnow A, Urbanetto JS, Greco PBT, Beltrame M, Luz EMF. Relationship between work ability in nursing and minor psychological disorders. Texto Contexto Enferm [Internet]. 2015 [cited 2021 Feb 23]; 24(2):362-70. DOI: http://dx.doi.org/10.1590/0104-07072015002580013.

15. Rodrigues DDM, Aquino RL, Antunes DE, Costa MM, Oliveira PC, Aragão AS. Work ability assessment for nursing team working at a large hospital in the region of Triângulo Mineiro - MG. Rev. Min. Enferm. [Internet]. 2019 [cited 2021 Feb 23]; 23:e-1260. DOI: http://www.dx.doi.org/10.5935/1415-2762.20190108.

16. Raboud J, Su DS, Burchell NA, Gardner S, Walmsley S, Bayoumi A, et al. Representativeness of an HIV cohort of the sites from which it is recruiting: results from the Ontario HIV Treatment Network (OHTN) cohort study. BMC Med Res Methodol [Internet]. 2013 [cited 2021 Feb 23]; 13:31. DOI: https://doi.org/10.1186/1471-2288-13-31. 